\title{
Non-steroidal anti-inflammatory drugs and the gastrointestinal tract
}

\author{
Authors: Foong Way David Tai ${ }^{A}$ and Mark E McAlindon ${ }^{B}$
}

\begin{abstract}
Non-steroidal anti-inflammatory drugs (NSAIDs) are used commonly but can cause foregut symptoms, peptic ulcer disease and small bowel enteropathy. Such iatrogenic injury can be complicated by gastrointestinal bleeding and perforation. Limiting NSAID use or co-administration with proton pump inhibitors (PPIs) reduce dyspepsia, peptic ulcer disease and rates of complications. Selective cyclo-oxygenase (COX)-2 inhibitors are as effective as adding PPIs in preventing upper and lower gastrointestinal complications. COX-2 inhibitors are suggested in those with high cardiovascular risk and the addition of PPI in those with high risk of bleeding. Where required, COX-2 inhibitor monotherapy may be preferred in unexplained iron deficiency anaemia.
\end{abstract}

\section{Introduction}

Non-steroidal anti-inflammatory drugs (NSAIDs), beneficial for their anti-inflammatory and analgesic effects, account for $8 \%$ of prescriptions worldwide and are used most commonly in those over the age of 65 years. Furthermore, there has been an increase in over the counter use with $26 \%$ using more than the recommended dose, and many undisclosed to medical professionals. ${ }^{1,2}$ Symptomatic upper gastrointestinal (GI) peptic ulcer disease and bleeding are the most recognised adverse events related to NSAIDs. However, complications related to NSAIDs can occur with or without symptoms, in the presence or absence of mucosal injury, and in the upper (to second part of the duodenum (D2)), mid- and lower gastrointestinal (GI) tract. In this article, we discuss the GI complications of NSAIDs, their presentation, risk factors and strategies to limit their occurrence.

\section{GI complications of NSAIDs and their presentation}

NSAIDs are second to Helicobacter pylori infection in causing peptic ulceration in the upper GI tract. They cause mucosal injury due to cyclo-oxygenase (COX)-1 inhibition by reduction of cytoprotective mucosal prostaglandins and reduction of the secretion of a protective bicarbonate mucus barrier in the stomach and small bowel.

A third of patients consuming NSAIDs develop foregut symptoms of dyspepsia (epigastric discomfort, bloating, post-prandial

Authors: ${ }^{\text {A }}$ clinical research fellow, Royal Hallamshire Hospital, Sheffield, UK; ${ }^{B}$ consultant gastroenterologist, Royal Hallamshire Hospital, Sheffield, UK nausea, early satiety and belching) and gastroesophageal reflux (heartburn and regurgitation). Such symptoms are not predictive of mucosal injury with $20 \%$ of symptomatic patients having a normal oesophagogastroduodenoscopy (OGD). ${ }^{3}$ However, up to $70 \%$ of patients with long term NSAID ingestion have endoscopic abnormalities (mucosal erosions, ulceration and subepithelial haemorrhage) despite only $10 \%$ complaining of dyspeptic symptoms. ${ }^{3}$ Serious peptic ulcer complications (such as bleeding and perforation) can therefore occur with no prior warning symptoms. Complications of gastroduodenal peptic ulcers occur up to five times more frequently among those consuming NSAIDs. ${ }^{4}$

Peptic ulcer bleeding occurs when the ulcer erodes into a subepithelial blood vessel. Presentation ranges from oozing from smaller calibre mucosal capillaries to more brisk bleeding from larger calibre arterial branches. Clinically, patients present with melaena, haematochezia and/or haematemesis, a fall in

\section{Key points}

NSAIDs can cause mucosal injury in upper, mid- and lower GI tract resulting in bleeding which can be overt (with melaena) or occult (iron deficiency).

Dyspepsia and reflux poorly predict the presence or absence of NSAID-induced peptic ulcer disease and its complications.

Use of NSAIDs, particularly with PPI co-administration, can cause small bowel mucosal injury and occult bleeding.

Co-administration of non-selective NSAIDs and PPIs or changing to selective COX-2 inhibitors can improve symptoms and reduce occurrence of complications of peptic ulcer disease in those at higher risk.

Selective COX-2 inhibitors alone should be considered in unexplained iron deficiency anaemia and co-administration with a PPI should be considered in patients with previous peptic ulcer complications.

KEYWORDS: non-steroidal anti-inflammatory drugs, NSAIDs, gastrointestinal, PPIs, COX-2 inhibitors

DOI: 10.7861/clinmed.2021-0039 
haemoglobin and rising urea as blood is digested to protein and metabolised to urea within the liver. Hypovolaemic shock is not uncommon, especially in cases with a delayed presentation or sudden erosion into a prominent vessel.

Shallow mucosal erosions and ulceration can also result in bleeding of the upper, mid- and lower GI tract causing iron deficiency and anaemia. Deep ulceration not otherwise presenting as bleeding, can also be complicated by perforation. Perforated peptic ulcers secondary to NSAIDs occur more commonly in the stomach than duodenum, and are associated with higher than recommended doses of NSAIDs. Chronic inflammation secondary to NSAID use can result rarely in small bowel obstruction secondary to diaphragm formation. These diaphragms are short web-like fibrostenotic segments of the small bowel which are not easily seen on computed tomography and classically described with NSAIDs, which can also, however, cause longer strictures indistinguishable from those due to Crohn's disease. Patients often present with intermittent cramping abdominal pain and anorexia over a period of time, with a distant history of NSAID use.

\section{Risk factors for NSAID-related bleeding and perforation}

The risk factors for bleeding secondary to NSAID-induced peptic ulceration are illustrated in Box 1. The relative risk (RR) of upper GI bleeding or perforation depends on the type of NSAID. Selective COX-2 inhibitors are less toxic to the GI tract (RR 1.9) than nonselective NSAIDs, with ibuprofen generally safer (RR 2.7) and more harmful are diclofenac (RR 4.0) or naproxen (RR 5.6). ${ }^{5}$ Patients with previous peptic ulcer bleeding or perforation are at the highest risk of bleeding. Infection with $\mathrm{H}$ pylori has a synergistic effect on risk of peptic ulcer bleeding among NSAID users, increasing risk of bleeding by 1.2 -fold. ${ }^{6}$ Other drugs also have a synergistic effect on bleeding risk. In particular, patients on dual antiplatelet therapy post-myocardial infarction (MI) and an NSAID have a two-fold increased risk of GI bleeding, and a 1.4-fold concomitant increased risk of adverse cardiovascular events (death, MI and stroke)

\section{Box 1. Risk factors for non-steroidal anti-} inflammatory drug gastrointestinal complications

Age $\geq 65$ years

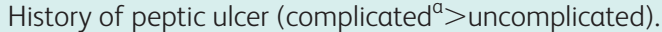

Concomitant therapy with antiplatelet agent, anticoagulants, corticosteroids and SSRIs.

Severe illness.

Helicobacter pylori infection.

Class of NSAID:

ns-NSAID > COX-2 inhibitor

naproxen $>$ indomethacin $>$ diclofenac $>$ ibuprofen $>$ rofecoxib $^{b}$

$>$ celecoxib.

High dose of NSAIDs.

Chronic use of NSAIDs.

${ }^{a}=$ complicated (bleeding or perforated) peptic ulcer disease are at highest risk of rebleeding; ${ }^{\mathrm{b}}=$ withdrawn from market; COX = cyclo-oxygenase; ns = non-selective; NSAID = non-steroidal anti-inflammatory drug; SSRIs = selective serotonin reuptake inhibitors. compared with those not on an NSAID. ${ }^{7}$ In this group of patients, NSAIDs should therefore be avoided.

\section{Managing and preventing complications of NSAIDs}

Limiting duration of NSAID use whenever possible is recommended. An OGD is warranted in patients on NSAIDs with dyspepsia over the age of 55 , or in younger patients with concomitant alarm symptoms (dysphagia or weight loss) to exclude oesophagogastric malignancy, irrespective of NSAID use, as per the National Institute for Health and Care Excellence guidance. ${ }^{8}$ In younger patients without alarm symptoms, a non-invasive test for $\mathrm{H}$ pylori, a trial of proton pump inhibitors (PPIs) and stopping NSAIDs where possible is reasonable. Patients presenting with GI bleeding or an acute abdomen should be questioned about dose and duration of NSAID use, past and present.

When long-term NSAID use is required, complications of NSAIDs can be prevented by co-administration of a PPI and/or changing a non-selective NSAID to a selective COX-2 inhibitor. Among patients with troublesome symptoms only, combination therapy of a traditional non-selective NSAIDs and PPIs reduce the incidence of dyspepsia by two-thirds compared with a $12 \%$ reduction following a switch to a selective COX-2 inhibitor. ${ }^{9}$ PPI co-administration with a non-selective NSAID reduces iatrogenic peptic ulcers in the upper GI tract and the risk of upper GI bleeding. $^{10,11}$ This should be considered in patients at risk of peptic ulcer bleeding (Box 1). The use of a selective COX-2 inhibitor, however, is also equally effective in minimising the risk of ulcer bleeding and, therefore, either strategy would be reasonable in those at risk of peptic ulcer disease. ${ }^{12}$

Patients with a history of ulcer bleeding or perforation are, however, at the highest risk of NSAID-related (re-)bleeding and, as such, a decision to prescribe NSAIDs should be considered carefully. In patients with previous ulcer bleeding, a combination therapy of a selective COX-2 inhibitor and PPI can further reduce recurrent upper GI ulcer bleeding risk compared to monotherapy with COX-2 inhibitors, particularly if low dose aspirin is also required to improve cardiovascular risk. ${ }^{13}$

To complicate matters further, the long-term use of non- and COX-2-selective NSAIDs may be associated with an increased risk of cardiovascular events. ${ }^{14}$ However, unlike rofecoxib, which was withdrawn for an unacceptable cardiovascular profile, there were no differences in cardiovascular adverse events (death, nonfatal MI or stroke) with celecoxib when used in moderate doses (up to 200 mg daily) compared with naproxen or ibuprofen. ${ }^{15}$ Furthermore, in patients who have had a previous peptic ulcer bleed, but requiring aspirin for cardiovascular prophylaxis and an NSAID, the CONCERN study suggests rates of rebleeding are lower with celecoxib compared with naproxen. ${ }^{13}$

\section{NSAIDs and the small bowel}

Distal to the second part of the duodenum, small bowel mucosal injury (erosions, ulceration and mucosal haemorrhage) is seen in up to $70 \%$ consuming NSAIDs and undergoing video capsule endoscopy. ${ }^{16}$ Such mucosal injury can lead to occult small bowel bleeding and NSAID consumption is suspected to be the cause in $10-15 \%$ of patients with iron deficiency anaemia (Fig 1). ${ }^{17}$ Selective COX-2 inhibitors seem to cause less small bowel mucosal injury than non-selective NSAIDs. However, because of gut dysbiosis, the combination of PPIs and COX-2-selective agents 


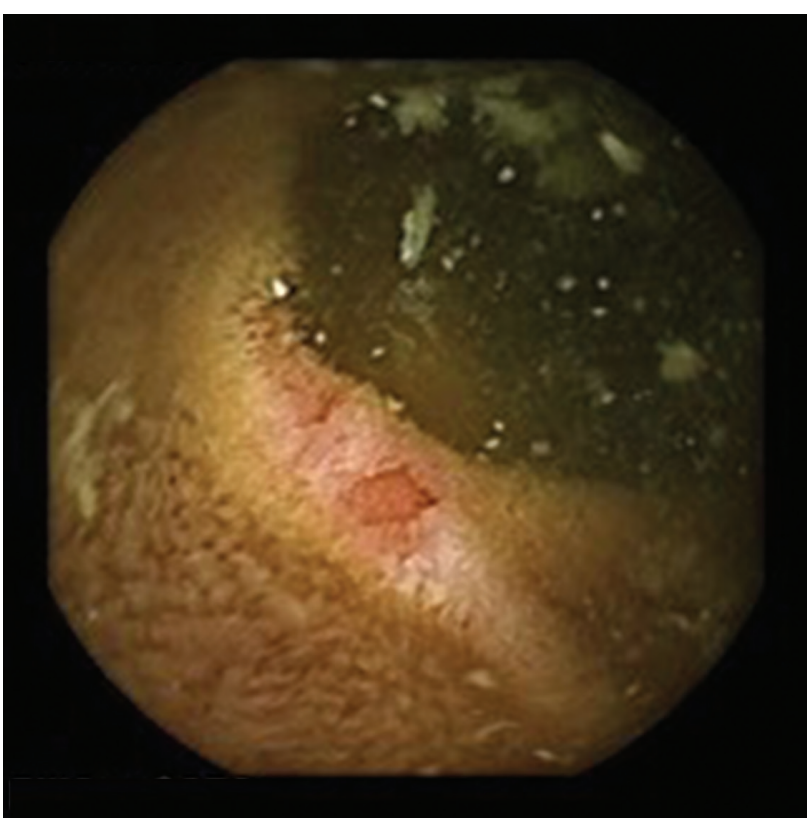

Fig 1. Small bowel mucosal injury on capsule endoscopy: mucosal erythema and erosion secondary to non-steroidal anti-inflammatory drugs.

have a detrimental effect on the small bowel, with increasing frequency and severity of small bowel ulcers compared with a cox-2 inhibitor alone. ${ }^{16}$

Clinical trials including anaemia (a fall in haemoglobin $>2 \mathrm{~g} / \mathrm{dL}$ ) as part of a composite endpoint with overt upper and lower GI bleeding and perforation show that, in trial patients taking NSAIDs, over $50 \%$ became anaemic without signs of overt GI bleeding and over $60 \%$ of these patients had no causative lesion identified on OGD. ${ }^{18}$ Significantly more patients who developed anaemia were randomised to a non-selective NSAID and PPI compared with COX-2 inhibitor monotherapy. ${ }^{18}$ This may, therefore, suggest that small bowel bleeding is a common cause of anaemia in patients taking NSAIDs, and may be more prevalent in those taking NSAIDs and concomitant PPI for gastroprotection. Selective COX-2 inhibitor monotherapy may, therefore, be a preferred strategy in those with unexplained iron deficiency anaemia or who develop iron deficiency while on a non-selective NSAID. ${ }^{19}$

\section{NSAIDs and microscopic colitis}

Exposure to NSAIDs and PPIs increases the risk of developing microscopic colitis (odds ratio 1.86 and 3.37, respectively). ${ }^{20}$ In patients with a chronic non-bloody watery diarrhoea, mucosal biopsies of the colon demonstrate a chronic inflammatory process of the lamina propria. Concomitant use of NSAIDs and PPIs and chronic exposure in between 4 and 12 months further increases the risk of microscopic colitis. It is therefore advised to discontinue these medications in this setting, where possible.

\section{Summary}

NSAIDs are a common cause of peptic ulcer disease in the stomach and duodenum, and dyspeptic symptoms occur commonly in the absence of peptic ulcer disease. Both are effectively treated by stopping NSAIDs, if possible, or using PPI therapy. Risk factors for bleeding include class, duration and dose of NSAID, concomitant drug therapies like antiplatelet agents and presence of $\mathrm{H}$ pylori infection. Selective COX-2 inhibitor monotherapy is an alternative to concomitant non-selective NSAIDs and PPI in protecting the upper GI tract from peptic ulcers and bleeding. It is recommended in patients who are at high risk of cardiovascular events and in combination with PPIs in patients who have previously had complicated gastroduodenal peptic ulcer disease. COX-2 inhibitor monotherapy may be more appropriate in unexplained iron deficiency anaemia given the risk of small bowel mucosal injury with NSAIDs and PPIs. Chronic and concomitant use of NSAIDs and PPIs increase the risk of microscopic colitis.

\section{References}

1 Zhou Y, Boudreau DM, Freedman AN. Trends in the use of aspirin and nonsteroidal anti-inflammatory drugs in the general US population. Pharmacoepidemiol Drug Saf 2014;23:43-50.

2 Sidhu R, Brunt LK, Morley SR, Sanders DS, McAlindon ME. Undisclosed use of nonsteroidal anti-inflammatory drugs may underlie small-bowel injury observed by capsule endoscopy. Clin Gastroenterol Hepatol 2010;8:992-5.

3 Larkai EN, Smith JL, Lidsky MD, Graham DY. Gastroduodenal mucosa and dyspeptic symptoms in arthritic patients during chronic nonsteroidal anti-inflammatory drug use. Am J Gastroenterol 1987;82:1153-8.

4 Garcia Rodriguez LA, Jick H. Risk of upper gastrointestinal bleeding and perforation associated with individual non-steroidal antiinflammatory drugs. Lancet 1994;343:769-72.

5 Massó González EL, Patrignani P, Tacconelli S, García Rodríguez LA. Variability among nonsteroidal antiinflammatory drugs in risk of upper gastrointestinal bleeding. Arthritis Rheum 2010;62:1592-601.

6 Huang JQ, Sridhar S, Hunt RH. Role of Helicobacter pylori infection and non-steroidal anti-inflammatory drugs in peptic-ulcer disease: a meta-analysis. Lancet 2002;359:14-22.

7 Schjerning Olsen AM, Gislason GH, McGettigan P et al. Association of NSAID use with risk of bleeding and cardiovascular events in patients receiving antithrombotic therapy after myocardial infarction. JAMA 2015;313:805-14.

8 National Institute for Health and Care Excellence. Gastrooesophageal reflux disease and dyspepsia in adults: investigation and management: Clinical guideline [CG184]. NICE, 2014.

9 Spiegel BM, Farid M, Dulai GS, Gralnek IM, Kanwal F. Comparing rates of dyspepsia with Coxibs vs NSAID+PPI: a meta-analysis. Am J Med 2006:119:448.e27-36.

10 Scheiman JM, Yeomans ND, Talley NJ et al. Prevention of ulcers by esomeprazole in at-risk patients using non-selective NSAIDs and COX-2 inhibitors. Am J Gastroenterol 2006;101:701-10.

11 Lanas A, García-Rodríguez LA, Arroyo MT et al. Effect of antisecretory drugs and nitrates on the risk of ulcer bleeding associated with nonsteroidal anti-inflammatory drugs, antiplatelet agents, and anticoagulants. Am J Gastroenterol 2007;102:507-15.

12 Wang $X$, Tian HJ, Yang HK, Wanyan P, Peng YJ. Meta-analysis: cyclooxygenase-2 inhibitors are no better than nonselective nonsteroidal anti-inflammatory drugs with proton pump inhibitors in regard to gastrointestinal adverse events in osteoarthritis and rheumatoid arthritis. Eur J Gastroenterol Hepatol 2011;23:876-80.

13 Chan FK, Wong VW, Suen BY et al. Combination of a cyclo-oxygenase-2 inhibitor and a proton-pump inhibitor for prevention of recurrent ulcer bleeding in patients at very high risk: a double-blind, randomised trial. Lancet 2007;369:1621-6. 
14 Trelle S, Reichenbach S, Wandel S et al. Cardiovascular safety of non-steroidal anti-inflammatory drugs: network meta-analysis. BM] 2011;342:c7086

15 Nissen SE, Yeomans ND, Solomon DH et al. Cardiovascular safety of celecoxib, naproxen, or ibuprofen for arthritis. $N$ Engl J Med 2016;375:2519-29.

16 Tai FWD, McAlindon ME. NSAIDs and the small bowel. Curr Opin Gastroenterol 2018:34:175-82.

17 Goddard AF, James MW, McIntyre AS, Scott BB, British Society of Gastroenterology. Guidelines for the management of iron deficiency anaemia. Gut 2011;60:1309-16.

18 Chan FK, Lanas A, Scheiman J, Berger MF, Nguyen H, Goldstein JL. Celecoxib versus omeprazole and diclofenac in patients with osteoarthritis and rheumatoid arthritis (CONDOR): a randomised trial. Lancet 2010;376:173-9.
19 Szeto CC, Sugano K, Wang JG et al. Non-steroidal anti-inflammatory drug (NSAID) therapy in patients with hypertension, cardiovascular, renal or gastrointestinal comorbidities: joint APAGE/APLAR/APSDE/APSH/APSN/PoA recommendations. Gut 2020;69:617-29.

20 Verhaegh BP, de Vries F, Masclee AA et al. High risk of druginduced microscopic colitis with concomitant use of NSAIDs and proton pump inhibitors. Aliment Pharmacol Ther 2016;43:1004-13.

Address for correspondence: Dr David Tai, Room P18, Royal Hallamshire Hospital, Sheffield Teaching Hospital NHS Foundation Trust, Glossop Road, Broomhall, Sheffield S10 2JF, UK

Email: david.tai@nhs.net 